\title{
Pero... ¿en qué año se licenció en Filosofía José Luis L. Aranguren?
}

\section{But... When did José Luis L. Aranguren obtain a Bachelor Degree in Philosophy?}

VÍCTOR MÉNDEZ BAIGES*

\begin{abstract}
Resumen: Fuentes autorizadas proporcionan las fechas 1936 y 1951 para la licenciatura y el doctorado en Filosofía de José Luis L. Aranguren (1909-1996). Sin embargo, ninguna de las dos es correcta. En este artículo se examina el expediente académico de Aranguren a fin de entender por qué esas fechas han ocupado el lugar de las que figuran en él: 1953 y 1954.

Palabras clave: Aranguren; filosofía española; historia de la universidad.
\end{abstract}

\begin{abstract}
Authoritative sources cite 1936 and 1951 as the dates for José Luis L. Aranguren's (1909-1996) bachelor degree in Philosophy and his doctorate in Philosophy. But neither date is accurate. The real dates are 1953 and 1954, as per his academic transcript, which this paper examines with a view to bringing out why the former pair of dates has taken the place of the latter.

Keywords: Aranguren; Spanish Philosophy; History of Universities.
\end{abstract}

La perentoriedad que se desprende del título de este artículo no descansa en el supuesto de que el año en que alguien obtuvo un grado universitario constituye un dato especialmente relevante. Tampoco en la idea de que eso valga para el caso de José Luis L. Aranguren. Quiere llamar la atención no sobre el dato en sí, sino sobre nuestra percepción de él. Pues estamos hablando de uno de los filósofos españoles más conocidos, de un influyente profesor de la Universidad, de un intelectual muy popular, acaso el más popular, en la España de finales del siglo XX. ¿Y qué ocurre con el año de su licenciatura? ¿Qué justifica el "pero" que encabeza estas líneas? Pues que no resulta nada fácil de averiguar. Tal dificultad dice algo acerca de la filosofía española y, por eso, vamos a tratar aquí de esa cuestión.

Recibido: 29/03/2017. Aceptado: 03/07/2017.

* Profesor titular de Filosofía del Derecho en la Universidad de Barcelona (victormendez@ub.edu). En relación con la historia de la filosofía española ha publicado recientemente "En los cuarenta años de La razón sin esperanza. Sobre Javier Muguerza, Francisco Vázquez y algunas cuestiones de Ética y Sociología” (en Astrolabio. Revista internacional de filosofía, año 2016, núm. 18), así como "La profesión de fe del filósofo", en el libro colectivo La voluntad de coherencia. Escritos en homenaje a José Manuel Bermudo, Horsori, Barcelona, 2016. 


\section{1. ¿Se licenció Aranguren en Filosofía en 1936?}

En principio, no parece difícil establecer cuándo alguien como Aranguren se licenció en Filosofía. Bastaría con consultar las fuentes autorizadas: sus obras completas, las biografías dedicadas al personaje, los estudios sobre su obra. En este caso contamos además con libros de homenaje, catálogos de exposiciones oficiales... Incluso con un "Archivo José Luis López Aranguren" en el CSIC.

La primera búsqueda en este tipo de fuentes arroja inmediatamente una fecha: 1936. Los seis volúmenes de sus obras completas, por ejemplo, cuentan todos con una cronología del autor que, para el año 1936, reza: "Licenciatura en Filosofía y Letras", y, para 1951, "Doctorado en Filosofía"1. Lo mismo afirma la breve biografía que proporciona la página web del Archivo José Luis López Aranguren del CSIC: “cursó estudios en el colegio de los Jesuitas de Chamartín de la Rosa y en 1928 comenzó estudios de Derecho en la Universidad Central de Madrid, donde obtuvo la licenciatura en 1931 y la licenciatura en Filosofía y Letras en 1936. En 1951 se doctoró en Filosofía"2.

El catálogo de la exposición Aranguren. Filosofía en la vida y vida en la filosofía, organizada en 2009 por el Instituto de Filosofía del CSIC, la Sociedad Estatal de Conmemoraciones Culturales y la Comunidad Autónoma de Madrid, también ofrece una cronología. Según esta, Aranguren se licenció en Filosofía y Letras en 1936, y se doctoró en Filosofía en $1951^{3}$.

Lo mismo aseguran biografías, monografías y libros de homenaje. El libro de Feliciano Blázquez José Luis López Aranguren. Medio siglo de la historia de España, encabezado por una nota del protagonista que lo declara una "perfecta biografía intelectual mía", contiene una cronología que, para 1936, señala: "Licenciatura en Filosofía y Letras”, y, para 1951, "Doctorado en Filosofía con la tesis El protestantismo y la moral" ${ }^{4}$. Otros libros acerca de Aranguren, como los publicados por Cristina Hermida del Llano ${ }^{5}$, o el titulado Retrato de José Luis L. Aranguren, de Eduardo López-Aranguren, Javier Muguerza y José María Valverde, recogen idénticas fechas ${ }^{6}$.

No parecería necesario indagar más. Aranguren se licenció en Filosofía en 1936. ¿Por qué dudar de esto?

Una primera razón es que la difusión del dato es bastante reciente. No hay ninguna referencia a él en las solapas o contraportadas de ningún libro de Aranguren. En algunas de ellas

1 Aranguren, J.L.L. (1994-1997), Obras completas, Madrid: Trotta. La cronología aparece en cada volumen, entre la introducción del editor y el prólogo del autor.

2 http://biblioteca.cchs.csic.es/archivos/aranguren/aranguren_biografia.php (consultado el 20/04/2017).

3 "Cronología", en VV.AA. (2009), Aranguren. Filosofía en la vida y vida en la filosofía, Madrid: Ministerio de Cultura, pp. 365-372.

4 Blázquez, F. (1994), José Luis López Aranguren. Medio siglo de la historia de España, Madrid: Editorial Ethos. La nota (pág. 1) dice "Es admirable, querido Feliciano, que hayas redactado esta perfecta biografía intelectual mía. Claro, para eso eres el mejor conocedor que existe de mi obra. Y que hayas dado a tu libro un enfoque más bien bibliográfico que estrictamente biográfico es lo que esperan quienes quieren oír hablar de mí. Muchas gracias y un gran abrazo".

5 Hermida del Llano, C. (1997) J.L.L. Aranguren, Estudio sobre su vida, obra y pensamiento, Madrid: Universidad Carlos III-Dykinson; y (1997) Aranguren (1909-1996), Madrid: Ediciones del Orto.

6 López-Aranguren, E., Muguerza J. y Valverde, J. Ma (1993), Retrato de José Luis L. Aranguren, Madrid: Círculo de Lectores-Galaxia Gutemberg. "Cuadro cronológico", pág. 92. 
el autor es descrito como "Doctor en Filosofía", pero siempre sin mención de fecha7. Hay una excepción: Las contraportadas de dos de sus libros más difundidos de los años setenta, Conversaciones con José Luis L. Aranguren y Talante, juventud y moral. Ambas aseguran que el autor es "Doctor en Filosofía y Letras con el libro La filosofía de Eugenio d'Ors (1945)" .8 Muchos repitieron después este dato, que resulta compatible con una licenciatura en $1936^{9}$.

El propio Aranguren nunca puso fecha concreta a la obtención de su licenciatura en Filosofía. En los primeros tiempos de su carrera evitó hablar sobre ello. En los años cincuenta se presentaba como un discípulo de Xavier Zubiri, asiduo a los cursos privados que este impartía, y eso le parecía suficiente. Es bien representativo lo que ocurre en una entrevista de 1958. Cuando el entrevistador se dirige al catedrático "que tan eminente lugar ocupa ya en la intelectualidad española" a fin de averiguar algo sobre su "formación intelectual", no consigue mucho: "Aranguren se turba un poco. Le resulta violento hablar de sí mismo. Apenas si logro sacarle que es de linaje vasco, como su apellido pregona..."10.

No fue mucho más explícito en su autobiografía intelectual, publicada en 1969 con el título de Memorias y esperanzas españolas. Habló allí de su formación universitaria, pero sin referirse a ningún estudio concreto. Señaló que, aunque su paso por la Universidad le proporcionó claridad y disciplina intelectual, "no produjo en mí esa revolución que, según vi después, cuando ya había ocurrido, perentoriamente necesitaba". Su timidez le impidió vincularse a "alguno de los admirados maestros". Por otra parte, "los años de Universidad suelen ser, simultáneamente años de diversión, de descubrimiento y goce de los alicientes de la juventud". No fue hasta la Guerra Civil, asegura, cuando alcanzó la concentración necesaria para lograr "la asistencia a mi propia existencia"11.

Más adelante fue más locuaz. En un texto autobiográfico de 1993 proporcionó información más concreta12. Reconocía allí que descubrió "relativamente tarde" la filosofía; que fue tras terminar la carrera de Derecho cuando ingresó en la Facultad de Filosofía y Letras, donde, "no siendo un chiquillo ya sino mayor de edad, me comporté más bien como alumno libre, leyendo más que asistiendo con regularidad a clase”. Eso no le impidió notar

7 Catolicismo día tras día, de 1955, es donde figura por primera vez la indicación de que el autor es Doctor en Filosofía.

8 Aranguren, J.L.L. (1975), Talante, juventud y moral, Madrid: Ediciones Paulinas; Aranguren, J.L.L. (1976), Conversaciones con..., Madrid: Ediciones Paulinas. Aunque Aranguren figura como autor, no son libros enteramente suyos. El primero es una antología de textos de Aranguren a cargo de Feliciano Blázquez. El segundo es una recopilación de entrevistas con él, también realizada por Blázquez. Ambos libros llevan un epílogo de Aranguren. El texto de las contraportadas, idéntico, va sin firma.

9 En su biografía de Aranguren, Blázquez se lamentó de que "haya ido propagándose el error de que La filosofía de Eugenio d'Ors fue el texto de la tesis doctoral de Aranguren”, y hasta proporcionó una lista de los que habían colaborado en ello: Julio Rodríguez-Puértolas, J.C. Mainer, Norbert Bilbeny, José Jiménez Lozano o Ana María Moix (Blázquez, F., 1991, pp. 50 y 51.). Su gesto se parece algo al de quien tira la piedra y esconde la mano, pues fueron los libros de Ediciones Paulinas los que difundieron el dato.

10 Jorge Mañach, “'El intelectual tiene que atender a lo social, pero sin comprometer su independencia', dice J. L. López Aranguren”, La Habana, Diario de la Marina, 14/12/1958, pág. 20.

11 Aranguren, J.L.L. (1969), Memorias y esperanzas españolas, Madrid: Taurus, pág. 38.

12 Aranguren, J.L.L. (1993), "Filosofía en la vida y vida en la filosofía”, en Isegoría, núm. 7, 1993, pp. 5-22. El texto recoge la primera de las Conferencias Aranguren, organizadas por el Instituto de Filosofía del CSIC, de cuyo patronato Aranguren era presidente. 
que, durante los años treinta, la "Sección de Filosofía, completamente orteguiana, bajo el decanato de García Morente y con profesores tan excelentes como ellos y Zubiri y Gaos, era ejemplar, única". No deja de insistir, sin embargo, en que su verdadera comprensión de la filosofía comenzó más tarde, lo que le llevó a entrar, en 1942, “como becario, más bien honorario, en el Instituto de Filosofía del CSIC"13.

En una larga entrevista con Javier Muguerza, aparecida ese mismo año, Aranguren volvió a rememorar su etapa de formación ${ }^{14}$. Explicó que, en los años veinte, decidió que "no me apetecía ser ingeniero e iba a hacer Letras, concretamente Derecho". Por eso, "me encontré, pues, estudiando el curso de acceso universitario a Letras, que era común a Derecho y Filosofía". Recordó haber tenido en ese curso como profesor de Lógica a Julián Besteiro, con el cual más adelante, ya en la Facultad de Filosofía y Letras, "a lo que no iría hasta después de acabar Derecho, ya instaurada la República, en 1932”, llegó “a tener cierta amistad”, pues "vivía en la calle de Carranza y mi familia en la de Sagasta, lo que me permitía acompañarle hasta su casa cuando salíamos juntos de la Facultad a última hora de la mañana"15.

“¿Cómo y por qué tomó la decisión de estudiar filosofía?” pregunta el entrevistador. Y Aranguren responde que "Al terminar la carrera de Derecho, trabajé un año como pasante en un bufete de abogados, lo que no me resultó muy excitante que digamos... la Facultad de Filosofía de la Universidad Central ejercía sobre mí una seducción cada vez mayor"16. “Cómo era aquella facultad?”:

Era de veras espléndida, aunque yo estudiaba por libre y no llegué a implicarme plenamente en la vida de la Facultad. Tenga en cuenta que para mí la de Filosofía constituía una segunda carrera, empezada a una edad ligeramente superior a la media del alumnado. Las clases se impartían ya en la Ciudad Universitaria, mientras que mi familia se había trasladado entretanto a nuestra casa de la calle de Velázquez, de suerte que me era menos cómodo desplazarme. Y, sobre todo, había iniciado a partir del año treinta y cuatro un nuevo noviazgo con la que luego sería mi mujer. Por todo ello, mi asiduidad no era la de un estudiante corriente y sólo asistía a las clases que de hecho me interesaban, a lo que coadyuvaba no poco la flexibilidad del llamado Plan García Morente, que era realmente un plan de estudios magnífico. En rigor, cada quien podía programarlos como mejor le conviniera y elegir tanto las materias como sus propios profesores, y no había más que tres exámenes a lo largo de toda la carrera: uno de ingreso, que me ahorré por tener la licenciatura de Derecho, otro intermedio y el examen final, en los que nos examinaban representantes de las diversas áreas de estudio ${ }^{17}$.

13 Ibid pág. 6. Esa beca refuerza la idea de que ya era licenciado en Filosofía por esa época.

14 Muguerza, J., (1993), "Del aprendizaje al magisterio de la insumisión. Encuentro con José Luis L. Aranguren”, en López- Aranguren, E. et al, (1993), pp. 65-88. Texto reproducido en Isegoría núm. 15, 1997, pp. 55-91.

15 Muguerza, J., (1993), pp. 69 y 70.

16 Ibid pág. 70.

17 Ibid pp. 70-71. 
A continuación, viene una sentida evocación de aquel centro en el que, "incluso los no implicados a fondo como yo mismo teníamos conciencia de pertenecer a una cierta aristocracia intelectual, de formar parte de algo así como un club intelectualmente distinguido"18.

El texto tiene algunas ambigüedades ${ }^{19}$. Lo que queda claro es que el entrevistado no se implicó mucho en la Facultad. No dice nada sobre la licenciatura, aunque la fecha de 1932 (la carrera tenía entonces cuatro cursos), o la referencia a un examen final en el que "nos" examinaban, encajan con 1936.

Ahora bien, que Aranguren nunca mencionará esa fecha resulta significativo. Permite albergar ciertas dudas sobre ella, al igual que lo hace la existencia de los llamados "siete magníficos".

Se llama así, algo jocosamente, a los miembros de la única promoción que se licenció en Filosofía antes de la Guerra según el llamado plan García Morente. Puesto que ese plan de estudios entró en vigor en 1932, esa promoción es, precisamente, la de 1936. Está integrada por siete personas: Francisco Alvárez González, Emilio Benavent, Manuel Granell, Antonio Rodríguez Huéscar, Julián Marías, Manuel Mindán y Leopoldo Eulogio Palacios ${ }^{20}$. Y el problema es que nunca nadie ha contado en ella con Aranguren. Si lo contamos, serían ocho. Parece imposible: ¿Cómo no se dieron cuenta, siendo tan pocos?

De ahí que siempre se hayan detectado dudas aquí y allá respecto a esa licenciatura de 1936. Hombres y documentos de la filosofía española, por ejemplo, el monumental diccionario de Gonzalo Díaz Díaz, no acaba de aceptar la fecha. En su entrada referente a Aranguren dice que sus estudios de Filosofía estaban "a punto de concluir cuando estalló la Guerra Civil". No precisa cuándo concluyeron. Puesto que añade que el doctorado "lo obtendría en 1951", el lector debe imaginar para ello una fecha posterior a la guerra, aunque anterior a ese $a \tilde{n} 0^{21}$.

El año de la licenciatura está relacionado con el del doctorado, y ha de ser, necesariamente, anterior. Hasta ahora hemos visto que, para lo segundo, se han postulado 1945 y 1951. En los años sesenta, en medio de los acontecimientos que acabaron con la expulsión de Aranguren de la Universidad franquista, el periodista Rodrigo Royo ofreció otra fecha: 1954.

Aranguren ocupaba desde 1955 la cátedra de Ética de la Universidad de Madrid. En 1965, mientras se tramitaba el expediente que acabó con su expulsión, la prensa falangista arremetió contra el profesor que se había puesto de parte de los estudiantes. Rodrigo Royo, director de la Revista SP, publicó en esa línea un artículo en el que, además de tratar a

18 Ibid, pág. 71.

19 Por ejemplo, no parece que Besteiro viviera nunca en la calle Carranza, en cuyo número 20 sí que se encontraba un famoso edificio del partido socialista. Al proclamarse la República, Besteiro vivía, según Saborit, en la calle Miguel Ángel, bien lejos de la calle Carranza (Saborit, A., 1961, Julián Besteiro, Impresiones Modernas S.A., México D.F., pág. 32). Más adelante se mudaría a la calle Grijalba, más alejada aún de Carranza. No se acaban pues de situar bien esos paseos con Aranguren. Más aún si tenemos en cuenta que el profesor consiguió una excedencia en 1931 y no se reintegró a la docencia hasta el curso 1935-36, y que el "alumno por libre" que era Aranguren ya vivía en la calle Velázquez ese curso.

20 Algunos se integraron bien tras la Guerra civil, tal es el caso de Leopoldo Eulogio Palacios, catedrático de Lógica de la Universidad de Madrid desde 1944, o el de Manuel Mindán y Emilio Benavent, ambos sacerdotes. Peor lo tuvieron otros. Álvarez González y Manuel Granell hicieron carrera en universidades sudamericanas. Marías y Rodríguez Huéscar permanecieron en España, marginados de la Universidad.

21 Díaz Díaz, G. (1991), Hombres y documentos de la Filosofía española, vol. IV, Madrid: CSIC, pág. 742. 
Aranguren de "camarada de la Falange", aireando su fecha de ingreso (1937), baja (1947) y número de carnet en F.E.T. y de las JONS, se refería al asunto de sus estudios filosóficos. Según Royo, el licenciado en Derecho Aranguren:

sintió una súbita vocación por la enseñanza universitaria en 1953, matriculándose el 7 de mayo de ese año de todas las asignaturas de la licenciatura en Filosofía y Letras. No puede decirse que se trate de precocidad, ya que el Sr. Aranguren, nacido en 1909, contaba por entonces con 44 años de edad, pero sí constituye una demostración de potencia mental. El 19 de junio de 1953 se le expidió el título de licenciado en Filosofía y el 30 de abril de 1954, el de Dr. en Filosofía... Y ahí viene la gran duda que está circulando por los claustros y las aulas universitarias: ¿Hizo, efectivamente, el Sr. López Aranguren toda la carrera de Filosofía y Letras de un solo trago, examinándose y aprobando todas las asignaturas, o simplemente se matriculó y se le extendió después el título? ${ }^{22}$

Era una impugnación en toda regla del par 1936/1951 (que tampoco nadie había formulado todavía), junto con una propuesta alternativa: 1953/1954. Prescindiendo ahora de lo que de canallesco hubiera en la persona, las intenciones o los modales de Royo, ¿tenían sentido las fechas que proporcionaba? Aranguren no se dignó responder nada. Pero acaso haya que darle la razón al periodista. Porque la verdad es la verdad, dígala Agamenón o un porquero veterano de la división azul.

En realidad, son las fuentes citadas al principio las que están deseando decir esa verdad y conceder la prioridad al par 1953/1954. Pues, a pesar de lo que diga la biografía del portal web del Archivo Aranguren, si se entra en el catálogo digitalizado y se escribe "título de doctor", lo que aparece es un documento cuya fecha es 1954-06-07. Y si miramos, no en la cronología, sino en el apartado Obra expuesta del catálogo de la exposición Aranguren. Filosofía en la vida y vida en la filosofía, vemos que recoge estos dos documentos:

Expediente Académico para la Expedición de Título de Licenciado en Filosofía y Letras a favor de D. José Luis L. Aranguren, 19 de junio-18 de noviembre de 1953. ARCHIVO GENERAL DE LA ADMINISTRACIÓN, FONDO MINISTERIO DE EDUCACIÓN, 32/17126 (LEGAJO 14523, EXP. 37) ALCALÁ DE HENARES, MADRID.

Expediente Académico para la Expedición de Título de Doctor en Filosofía, sección Filosofía, a favor de D. José Luis L. Aranguren, 5 de mayo-3 de julio de 1954. ARCHIVO GENERAL DE LA ADMINISTRACIÓN, FONDO MINISTERIO DE EDUCACIÓN, 32/17563 (LEGAJO 14742, EXP. 149) ALCALÁ DE HENARES, MADRID $^{23}$.

22 Royo, R., "Las incógnitas éticas del profesor Aranguren”, Revista SP, 15/06/1965, pág. 29. Royo (1922-1982), "falangista de izquierdas", era un personaje bastante inclasificable.

23 VV.AA. (2009), pág. 391. 
Hasta el propio Feliciano Blázquez reconoce, en su introducción al primer volumen de las obras completas, que 1936 no es una fecha que haya que tomarse tan en serio. Pues escribe allí que Aranguren "estaba a punto de acabar" sus estudios en Filosofía ese año ${ }^{24}$. Como añade que se doctoró en 1951, deja sin aclarar cuándo obtuvo la licenciatura. Eso al lector del primer volumen, porque el de los otros cinco ha de leer que Aranguren se licenció en Filosofía en 1936. ¿Cómo debe entenderse esto?

Al igual que en el caso de aquel embajador en Portugal llamado Porras y Porras, aquí lo que molesta es la insistencia. ¿Por qué los responsables del Archivo Aranguren no consultan los propios documentos que custodian? ¿Por qué la cronología del catálogo de una exposición oficial no aprovecha la obra expuesta? ¿Por qué, tras reconocer en el primer volumen que el autor no se licenció en Filosofía en 1936, se da a continuación esa fecha por buena? ¿Qué tienen de malo los años posteriores a 1936 para situar en alguno de ellos la licenciatura? ¿Qué tiene de malo 1953? ¿Que es muy tardío? ¿Que obliga a abandonar 1951 como fecha del doctorado? ¿Que está demasiado cerca del año en que Aranguren ganó la cátedra de Ética?

Está claro que se manifiesta en todo esto una cierta tensión. La página sobre Aranguren del sitio web "Proyecto Filosofía en español" colabora de esta manera:

Doctor en Filosofía en 1954 por la Universidad de Madrid, con la tesis El protestantismo y la moral (243 h., T2286), leída el 10 de mayo de 1954. [La cronología de Aranguren que figura en el volumen 1 de sus Obras completas, Madrid 1994, págs. 17-20, asegura que obtuvo el doctorado en 1951, pero el Catálogo de Tesis Doctorales de la Universidad Complutense la data en 1954 y precisa la fecha de lectura que ofrecemos: 10-05-1954.]

De todo lo dicho quedan dos cosas claras. Aranguren se licenció en Filosofía en 1953, y se doctoró en 1954. También queda claro que algunos tienen ciertas dificultades en admitirlo.

\section{Aranguren se licenció en Filosofía en 1953}

A finales del siglo pasado comenzó a consolidarse una nueva disciplina llamada "Sociología de la Filosofía”, para la cual autores como Pierre Bourdieu o Randal Collins se convirtieron en referentes importantes. En España, esta disciplina que pretende estudiar el aspecto social de la producción filosófica ha encontrado un particular acomodo. El libro La Filosofía española. Herederos y pretendientes, de Francisco Vázquez, ha sido quizás el mejor exponente. En 2013, José Luis Moreno Pestaña publicó La norma de la filosofía. La configuración del patrón filosófico tras la Guerra Civil, dedicado a estudiar sociológicamente la filosofía de los primeros años del franquismo ${ }^{25}$.

24 Blázquez, F., en Aranguren, J.L.L. (1994), Obras completas, vol. 1, Madrid: Trotta, pág. 11. El mismo Aranguren, en un texto titulado "Esquema de una autobiografía", publicado en la revista Triunfo del 1 de noviembre de 1981, pp. 55-59, ya había indicado que, "a punto de acabar aquellos estudios [de Filosofía] estalló la Guerra Civil..." (pág. 57).

25 Vázquez García, F. (2009), La Filosofía española. Herederos y Pretendientes. Una lectura sociológica (19631990), Madrid: Abada Editores; Moreno Pestaña, J.L. (2013), La norma de la filosofía. La configuración del patrón filosófico tras la Guerra Civil, Madrid: Biblioteca Nueva. 
En los dos libros se trata de Aranguren. En el primero, como el pensador alrededor del cual se constituyó la principal red alternativa que dio lugar a la "transición filosófica". En el segundo, puesto que se trata de reconstruir redes y trayectorias de los años cuarenta y cincuenta, también se hace alguna referencia a él. De esta manera, atendiendo a un fenómeno de "aceleración" dentro del campo, Moreno Pestaña escribe:

Aranguren se había licenciado en Derecho en 1932 y en Filosofía en 1953, después de aprobar en un solo año 10 asignaturas (las otras seis las había cursado en 19351936) y de superar el examen de grado de licenciado el 25 de octubre de 1953. En apenas siete meses (el 5 de mayo de 1954), y dirigido por el joven católico Millán Puelles, lee su tesis El protestantismo y la Moral ante un tribunal que la califica de sobresaliente por mayoría ${ }^{26}$.

Moreno Pestaña ha consultado los archivos. Y ofrece datos precisos: los de los expedientes que se guardan en el Archivo General de la Administración. Sus datos coinciden, aunque no en el detalle, con los de Royo. Da un nombre que figura en el expediente de doctorado y que, hasta donde sé, nadie había publicado hasta entonces: el del director de tesis.

Antonio Millán Puelles (1921-2005) no era el nombre que cabía esperar. Doce años más joven que Aranguren, doctor en 1947, catedrático de Fundamentos de Filosofía en la Universidad de Madrid desde 1951, era el benjamín del grupo intransigente que venía dominando la sección de Filosofía madrileña desde el final de la Guerra Civil, grupo en el que también militaban Rafael Calvo Serer y Leopoldo Eulogio Palacios. Vinculado a la editorial Rialp, a la revista Arbor y al Departamento de Filosofía de la Cultura y de Culturas Modernas que Calvo dirigía en el CSIC, Millán era un producto bien típico de la época de las “opusiciones".

Si miramos al momento en que tiene lugar la licenciatura, octubre de 1953, la elección de director puede sorprender. Desde el nombramiento de Ruiz-Giménez como ministro de Educación Nacional dos años antes, se había venido desarrollando un enfrentamiento cada vez más abierto entre los partidarios y los detractores de la política de ese ministro. Ese enfrentamiento, polarizado en torno a las figuras de Laín Entralgo, rector de la Universidad de Madrid, y Calvo Serer, hombre fuerte de la revista Arbor, dio origen a una discusión en torno a la catolicidad del pensamiento español que se conoce como la polémica entre comprensivos y excluyentes. Dicha polémica, de la cual la cuestión acerca de la condición cristiana de la Filosofía era un elemento esencial, llegó a su cénit, precisamente, a mediados de 1953. A lo largo de ese año, un curso de homenaje a Ortega organizado por Laín, junto con los intentos del Ministerio de Educación para controlar al CSIC, donde se habían hecho fuertes sus oponentes, enconaron especialmente la pugna. Un libro de Calvo Serer aparecido en agosto, en el que se denunciaba la pretensión de resucitar "la corriente cultural derrotada y en decadencia" mediante la introducción de elementos extraños en la Sección de Filosofía, muestra hasta qué punto habían llegado las $\operatorname{cosas}^{27}$. En septiembre, la protesta desde medios

26 Moreno Pestaña, J.L, (2013), pág. 80.

27 Calvo Serer, R. (1953), La configuración del futuro, Madrid: Rialp, pág. 239. La cátedra de Metafísica de la Universidad de Madrid quedó vacante en 1953 por la jubilación de Ortega. Se dijo que Julián Marías tenía intención de presentarse. Sobre esa pretensión, aunque no mencione a Marías, quería alertar Calvo, quien ofrece en su libro una lista de los filósofos que se ajustarían al cargo. 
eclesiásticos ante la pretensión de la Universidad de Salamanca de celebrar un homenaje a Unamuno empeoró la situación.

Ese mismo mes, Rafael Calvo Serer dio la gran campanada de la polémica. Publicó en Francia un escrito decididamente político en el que proponía que, agotada la coalición entre "totalitarios" (Laín) y "demócrata cristianos" (Ruiz-Giménez), Franco se decidiera a encomendar el gobierno a una "tercera fuerza". Para mostrar el poder de esta, exhibía una larga lista de sus integrantes, entre los que se contaba Antonio Millán Puelles ${ }^{28}$.

La consecuencia de ese artículo fue un gran escándalo y que la autoridad invocada pusiera un fin abrupto al debate intelectual. Calvo fue destituido de todos sus cargos en el CSIC. La prensa falangista le cubrió de improperios. La revista Arbor cambió radicalmente de orientación. Escrivá de Balaguer visitó a Franco. Ruiz-Giménez cesó en sus intentos de controlar el CSIC. Y el enfrentamiento entre unos y otros quedó, como resultado, latente y en una especie de tablas.

De acuerdo con su expediente académico, es en plena efervescencia de esa polémica cuando tiene lugar la primera presencia de Aranguren en la Facultad de Filosofía y Letras. El 19 de junio de 1953 consta la matrícula de diez asignaturas para el curso 1952-1953: Filosofía, Historia de la Filosofía, Lengua y Literatura Latinas, Lengua Griega, Psicología, Lógica, Ética, Estética, Metafísica y Sociología ${ }^{29}$. Ese mismo documento hace constar que el alumno había ingresado en la Facultad el 20 de octubre de 1935. Se escribe al margen de este ingreso "Dispensado". El documento certifica que el alumno verificó el examen intermedio el 17 de junio de 1936 (al margen consta: "Admitido"), y le reconoce seis asignaturas del curso 1935-1936: Filosofía, Lógica, Estética, Lengua Griega, Lengua Latina e Historia del Arte.

¿Podía matricularse un alumno tan extemporáneamente, ya acabado el curso? Sí. Una Orden del Ministerio de Educación Nacional del 22 de junio de 1946 autorizaba al rector a decidir discrecionalmente sobre las solicitudes de matrícula formuladas fuera de plazo. Y el rector era Laín, el mismo que, según el propio Aranguren, le sugirió el 20 de abril de 1953 que se preparara para presentarse a una cátedra de Ética ${ }^{30}$. Las fechas encajan.

El alumno se ha matriculado de diez asignaturas, pues tenía seis ya superadas. El plan de estudios vigente para Filosofía, de 1944, establecía que la licenciatura constaba de dos cursos comunes más tres de especialidad. También ordenaba que en el expediente figurara la nota de cada asignatura. Pero aquí no hay calificaciones y solo dieciséis asignaturas. ¿Qué pasa?

Pues que el alumno está cursando la carrera según el Decreto para las Facultades de Filosofía y Letras de 1931 (Gaceta de Madrid del 16 de septiembre). Según ese decreto, la carrera de Filosofía y Letras se cursaba en cuatro años, uno común y tres de especialidad. De modo experimental, el decreto autorizaba a las facultades de Madrid y Barcelona a establecer un régimen especial. La de Madrid, ejerciendo esa autonomía, desarrolló un plan de estudios propio que entró en vigor en 1932, el llamado Plan García Morente.

28 Calvo Serer, R. (1953), "La politique interieur dans l'Espagne de Franco", en Écrits de Paris IX.

29 Este y el resto de los datos relativos a los estudios de Aranguren que viene a continuación están tomados de los expedientes académicos para la expedición del Título de Licenciado en Filosofía y Letras y para la Expedición de Título de Doctor en Filosofía que se custodian en el Archivo General de la Administración en Alcalá de Henares, ya citados.

30 Aranguren, J.L.L. (1969), pág. 92. 
Ese plan era bien singular y un verdadero experimento pedagógico. No había asignaturas obligatorias ni orden de prelación en la matrícula. La asistencia a clase no era obligatoria, y no había examen ni nota por asignatura. Únicamente había tres exámenes. El de ingreso, el cual, si no se aprobaba, exigía cursar un curso preparatorio; un examen intermedio, que el alumno podía realizar cuando lo considerase adecuado y que versaba sobre materias comunes (Lenguas, Introducción a la Filosofía, Literatura, Historia e Historia del Arte); y un examen final que daba derecho al título de licenciado. Para presentarse a este último había dos condiciones: haber estado matriculado en la facultad al menos tres cursos, sin contar el preparatorio; y haber estado matriculado en las materias objeto de examen, las cuales eran diferentes según el título al que se aspirara ${ }^{31}$. Todos los exámenes tenían una parte escrita $\mathrm{y}$ otra oral.

El sistema no implicaba falta de rigor. Era frecuente que los estudiantes esperaran más de un año para presentarse al examen intermedio. En los exámenes había muchos suspensos. Según Julián Marías, solo un 20\% de su promoción se licenció en 1936. Hubo incluso protestas al decano por este asunto.

En junio de 1953 se considera a Aranguren alumno de ese plan. Se le reconoce aprobado el examen intermedio, se le inscribe en diez asignaturas y, aunque solo consta como matriculado en la Facultad dos cursos, y no tres, como era preceptivo, se le considera apto para presentarse al examen final. Según su expediente, la parte escrita la realizó el 23 de octubre de 1953, con la calificación de "admitido". La parte oral tres días más tarde. Obtuvo la calificación de sobresaliente. Firman el acta cinco profesores. Uno de ellos es Millán Puelles.

Esto sucedía en plena tormenta posterior al artículo de Écrits de Paris. En principio, Aranguren, ligado a Laín y a la revista Cuadernos Hispanoamericanos, participante en el curso de homenaje a Ortega y autor que ha publicado en la editorial Revista de Occidente, es un comprensivo. Millán, muy ligado a Calvo, a Arbor y al CSIC, es un excluyente. Pero, como ha señalado muy bien Onésimo Díaz Hernández en su libro sobre Calvo Serer, no se deben exagerar las diferencias entre ambos grupos ${ }^{32}$. Todos colonizaban los organismos oficiales. Todos se sentían ligados a Franco y a la Guerra Civil. Todos iban, en definitiva, en el mismo barco. Millán y Aranguren eran, por lo demás, individuos moderados dentro de sus grupos. Ambos veían su trabajo intelectual como "acción católica" y se interesaban por la concordancia entre el pensamiento escolástico y el contemporáneo. Los dos consideraban extraordinario el trabajo filosófico de Zubiri.

Pero es que, además, estamos ya dentro del ciclo pacificador impuesto durante esos meses finales de 1953. En septiembre, el Ministerio convocó la cátedra de Metafísica de la Universidad de Madrid, rebautizada como "Ontología y Teología Natural”. En febrero de 1954, el BOE anunció el tribunal. Los tres vocales automáticos fueron Pedro Font Puig, Calvo Serer y Jaime Bofill. Los de designación libre por el Ministerio no pudieron ser más halagadores para la excluyente Sección de Filosofía madrileña: el dominico Santiago Ramírez, presidente, y Antonio Millán Puelles, secretario.

31 La guía docente del curso 1932-1933, y para el título de licenciado en Filosofía, menciona Historia de la Filosofía, Latín, Árabe o Griego, Psicología, Lógica, Ética, Estética, Metafísica, Psicología, Sociología y una materia a elección del estudiante.

32 Díaz Hernández, O. (2008), Rafael Calvo Serer y el grupo Arbor, Valencia: Universitat de València. 
Así que no debe extrañar que, una vez licenciado Aranguren, Millán se encargase de la dirección de su tesis doctoral. Eran tiempos de tregua, y se trataba simplemente de hacer un doctor en Filosofía. Nadie podía imaginar que el recién licenciado pudiera en breve ganar una cátedra. En marzo de 1954, cuando se convocó a los opositores a la cátedra de Ontología y Teología Natural, la ganó alguien bien próximo a la Sección de Filosofía madrileña: Ángel González Álvarez, el primero de la lista que había presentado Calvo en La configuración del futuro.

La tesis de Aranguren se leyó poco después, el 5 de mayo. En su expediente de doctorado, en el cuadro preparado para apuntar los cursos seguidos y las calificaciones obtenidas, está escrito: "Nota. Se tramita este expediente de acuerdo con el Decreto de 24 de agosto de 1932 (Gaceta del 27)".

Lo que esto quiere decir es que Aranguren no tenía que hacer los cursos de doctorado exigidos por el Decreto de Ordenación de la Facultad de 1944, porque se le aplicaba la legislación de doctorado de la época de la República. Y el decreto de doctorado de 1932 no preveía cursos para ese grado. Sólo exigía a) "ser licenciado"; b) "trabajar durante un curso completo, como mínimo, después de obtenida la licenciatura, bajo la dirección de un catedrático"; y c) la defensa de la tesis ante un tribunal de cinco profesores. Consciente de lo que significaba abandonar el viejo ordenancismo, el decreto apelaba al "honor y a la responsabilidad" de los claustros para organizar bien este asunto.

En el acta del grado de doctor se reconocen las firmas de los miembros del tribunal. Presidente: Santiago Montero Díaz; Secretario: Antonio Millán Puelles; Vocales: Rafael Calvo Serer, Leopoldo Eulogio Palacios y José María Sánchez de Muniaín. El primero y el último eran profesores alineados con el Ministerio ${ }^{33}$. Pero ha de notarse que la mayoría del tribunal la tienen los excluyentes.

La calificación que consta en el acta es Sobresaliente (por mayoría). Intuyo que fue Calvo Serer quien impidió la unanimidad. Aranguren, que la firma como alumno, es el de más edad de todos los implicados. Se ha licenciado y doctorado en menos de siete meses. Los que van de octubre de 1953 a mayo de 1954.

¿Era posible eso sin cometer irregularidades? La verdad es que no mucho. De acuerdo en que los alumnos podían acabar los grados ya comenzados según los planes antiguos. De acuerdo en que el rector podía admitir matrículas fuera de plazo y dispensar la escolaridad. Pero no está nada claro que, licenciándose en 1953, Aranguren pudiera cursar el doctorado según el decreto de 1932. Pues, implantados ya completamente los nuevos grados, una Orden del Ministerio de Educación Nacional de 30 de noviembre de 1948 (BOE del 2 de enero de 1949) estableció que el derecho de los licenciados por planes antiguos a matricularse de doctorado por planes antiguos, con la excepción de Medicina, caducaría el curso 1950-1951.

Mira por dónde nos aparece 1951. ¡La última oportunidad para matricular el doctorado en Filosofía para los licenciados del plan republicano! Ahora entendemos qué tiene de malo el par 1953/1954. Que, aunque no hubiera obstáculo a licenciarse en 1953 por el plan de

33 Santiago Montero Díaz era catedrático de Historia de la Universidad de Madrid. Fue uno de los profesores separados de su cargo en 1965. Sánchez de Muniaín fue Director General de Enseñanza Media con RuizGiménez. 
1932 si se había empezado a estudiar según él, si Aranguren matriculaba el doctorado en 1953 debía hacerlo obligatoriamente por el plan de 1944, examinándose, al menos, de los cursos previstos en este. Se decidió sin embargo doctorarle de acuerdo con el decreto de 1932, como si hubiera matriculado el doctorado antes de 1951.

No era la legislación aplicable. Y ni siquiera se molestaron en cumplirla. Pues, entre los escasos requisitos que, confiando en el honor y la responsabilidad de los claustros, estableció el decreto de 1932 figuraba el de "trabajar durante un curso completo como mínimo", exigencia no dispensable que Aranguren, entre octubre de 1953 y mayo de 1954, obviamente no cumplió.

El expediente muestra pues que estamos ante una componenda. No ha de extrañarnos. No era gente que se arredrara ante irregularidades ${ }^{34}$. En los años cuarenta, el plan de estudios de García Morente fue tratado como un espléndido botín de guerra, y la "aceleración" de las carreras fue la norma. Que se aviniese ahora a este tipo de irregularidades alguien como Calvo Serer solo muestra hasta qué punto Aranguren le parecía poco amenazador. Pues Calvo era muy intransigente con la integridad ideológica de todo lo relacionado con la Filosofía. Lo muestra bien su conducta en el asunto del doctorado de Julián Marías.

Julián Marías se licenció en 1936. En la confusión de 1939 obtuvo el premio extraordinario de licenciatura y, a principios de 1941, publicó una historia de la Filosofía que terminaba con un agradecimiento a Dios por haberle permitido poner fin a su obra con un nombre español: el de José Ortega y Gasset. No gustó mucho eso en la Sección de Filosofía. De ahí que, en enero de 1942, cuando presentó su tesis doctoral, el tribunal la calificara como suspenso. En la papeleta estaba impreso: "Las calificaciones serán sobresaliente o aprobado". Pero escribieron "suspenso" y así se quedó 35 .

En 1951, y ante el límite temporal puesto a los doctorados por la Orden del Ministerio, desde el decanato de la Facultad -no desde la Sección de Filosofía- se propuso a Marías que se doctorara. Estuvo de acuerdo si presentaba exactamente la misma tesis. Enterado Calvo Serer, elevó una instancia al decano denunciando la nulidad del acto de volver a juzgar lo ya declarado insuficiente. Hizo notar asimismo que no había transcurrido el año preceptivo entre la solicitud y la lectura de la tesis. Pero el decano tiró adelante y la tesis se leyó en julio de 1951. Calvo elevó un recurso al rector que quedó en nada ${ }^{36}$.

Nada de esta beligerancia la encontramos hacia Aranguren. Como máximo, Calvo se negó a otorgar el sobresaliente. Lo que esto revela es que, a sus ojos, no representaba "la corriente cultural derrotada" en 1936 y "en decadencia" desde entonces. No tenía nada que ver con ella.

Aranguren era efectivamente, en 1953/54, "uno de los nuestros”. De ahí que recibiera el trato correspondiente. No otra cosa muestra su expediente académico. Más adelante no ha gustado admitir esa realidad. Y se ha huido hacia la Facultad anterior a la Guerra, hacia el par 1936/1951. A eso precisamente es a lo que se refirió Javier Marías cuando, respecto

34 Una Orden del Ministerio de Educación Nacional de 22 de mayo de 1950 relativa a la firma de documentos exigía que, en las actas, los nombres y apellidos se escribieran al pie de las firmas. Pero nadie lo hace en el acta de doctorado de Aranguren, lo que ha supuesto un trabajo extra para el autor.

35 Marías, J. (2008), Una vida presente. Memorias, Madrid: Páginas de espuma, pp. 238-39.

36 Relatado en Díaz Hernández, O. (2008), pp. 337-341. 
a Aranguren, habló de "falsear el pasado", de "biografía-ficción" y de "biografías ficticias y maquilladas" 37 .

¿Qué nos muestra, en todo caso, la contraposición entre los pares 1936/1951 y 1953/1954? En primer lugar, la profunda distancia entre la filosofía española y su propia historia. Porque ¿cómo es posible que resulte tan difícil averiguar un dato tan sencillo y tan reciente? ¿No revela eso una cierta desidia general? Desidia acaso disculpada con la idea de no suministrar munición a los que son "de otra cuerda". Pero desidia que genera más desidia. Desidia indisculpable.

Y no se trata aquí solo de redes y trayectorias, que también. La obra y los principales temas de Aranguren, la relación del intelectual con la política, la asunción de la crisis religiosa y metafísica, la preocupación por la desmoralización de la sociedad, la apertura a la ética analítica... son cosas todas que no pueden entenderse sin referirse a la situación en la que se encontraban los intelectuales católicos en los años cincuenta. De ahí que el baile de fechas tenga más importancia de lo que, a simple vista, pueda parecer.

\section{Coda}

Cabe aun una cuestión: ¿Estuvo Aranguren matriculado en la Facultad de Filosofía y Letras durante el curso 1935/1936 y aprobó allí el examen intermedio?

Eso dice su expediente académico de 1953. Pero ya hemos visto que no es un documento muy fiable. Los recuerdos del propio Aranguren tampoco lo son. En sus memorias no dijo nada sobre haber estado matriculado en esa Facultad. Solo mucho más tarde se refirió a ello, nunca con excesiva precisión. En la entrevista de 1993 le hemos visto afirmar que a la Facultad de Filosofía y Letras "no iría hasta después de acabar Derecho, ya instaurada la República, en 1932”. ¿En 1932? Dejaba además claro que "estudiaba por libre y no llegué a implicarme plenamente en la vida de la Facultad", pues "mi asiduidad no era la de un estudiante corriente y sólo asistía a las clases que de hecho me interesaban".

¿Qué clases eran esas? Según su expediente, Filosofía, Lógica, Estética, Lengua Griega, Lengua Latina e Historia del Arte. Hay algo raro en ellas. ¿Por qué volvió a matricular Lógica, que ya había aprobado en el curso preparatorio de Derecho? Si cursó la asignatura "Introducción a la Filosofía”, ¿por qué nunca se refirió a ello? ¿No asistía? ¿Es que no le "interesaba" esa clase, aunque sí la de Lógica? ¿O es que acompañaba a "su casa” a Besteiro sin haber ido antes a sus clases? En el examen intermedio se preguntaba además sobre Introducción a la Filosofía, y no sobre Lógica. ¿Y por qué vuelve a constar esa "Filosofía" como matriculada para el curso 1952-1953, junto con Lengua Griega y Estética, todas ellas ya cursadas en 1935-36? ¿Y por qué matricula Aranguren Lógica para el curso 1952-1953, ahora por tercera vez? ¿Era eso admisible? Contar varias veces la misma asignatura hasta llegar a las dieciséis.

Por otro lado, ¿no llama la atención que un alumno tan poco asiduo aprobara el examen intermedio nada más acabar su primer curso? El expediente así lo afirma. Pero sabemos que no era lo corriente. Ni siquiera entre los estudiantes que asistían a clase. ¿No dice eso dema-

37 Javier Marías en El País, "Nada importa" (18/05/88), "El padre" (16/06/94), "El artículo más iluso" (26/06/1999). 
siado de la capacidad de alguien que se describió a sí mismo como devoto de las diversiones por esa época y "no implicado a fondo” en la Facultad?

En definitiva, ¿es posible que la componenda de 1953 llegara hasta el punto de inventar un primer curso y un examen intermedio aprobado para poner en marcha todo lo demás? Hemos visto que el propio Aranguren se consideraba a sí mismo estudiando Filosofía y Letras solo porque hizo el curso preparatorio de Derecho. De acuerdo con sus palabras, hacer Derecho y hacer Letras era prácticamente lo mismo: “iba a hacer Letras, concretamente Derecho. Al año siguiente me encontré, pues, estudiando el curso de acceso universitario a Letras, que era común a Derecho y Filosofía".

Rafael Lapesa nos ha dejado una evocación de aquel curso preparatorio desde el punto de vista de un estudiante de Filosofía y Letras. Las clases se impartían, cuenta, en "una única aula, oscura, pero capaz de dar asiento en sus bancos a los doscientos cincuenta estudiantes totalizados entre Filosofía y Letras, exigua minoría, y Derecho. Abundaban en aquella masa los señoritos, pueblerinos o urbanos, sin desbravar... En ocasiones el espectáculo de gamberrismo escolar era comparable al descrito por Baroja en alguna de sus más famosas novelas. Pero afortunadamente no se prolongaba en los cursos siguientes, ya privativos de nuestra facultad..." 38 .

¿No es posible que ese curso en "Letras, concretamente Derecho" fuera considerado suficiente por los no muy exigentes instructores del expediente de 1953? ¿Que lo asimilaran al primer curso de la Facultad de García Morente y le aplicaran la conveniente aceleración? Los que disponían en aquella época de la Facultad eran capaces de eso y de mucho más. Tampoco sería la mayor irregularidad que cometieron. De hecho, en algunos momentos cierro los ojos y los veo. Veo a aquellos señoritos, pueblerinos o urbanos, aún sin desbravar. Aunque lo mejor será que dejemos este asunto en puntos suspensivos...

\section{Referencias}

ARANGUREN, José Luis L. (1994-1997): Obras completas, 6 vols., Trotta, Madrid.

ARANGUREN, José Luis L. (1968): Memorias y esperanzas españolas, Taurus, Madrid.

ARANGUREN, José Luis L. (1975): Talante, juventud y moral, Ediciones Paulinas, Madrid.

ARANGUREN, José Luis L. (1976): Conversaciones con..., Ediciones Paulinas, Madrid.

ARANGUREN, José Luis L. (1981):"Esquema de una autobiografía”, en Triunfo núm. 13, año XXXV, pp. 55-59.

ARANGUREN, José Luis L. (1993): "Filosofía en la vida y vida en la filosofía", en Isegoría núm. 7, 1993, pp. 5-22.

BLÁZQUEZ, Feliciano (1994): José Luis López Aranguren. Medio siglo de la historia de España, Editorial Ethos, Madrid.

BLÁZQUEZ, Feliciano (1994): "Introducción. José Luis L. Aranguren. El hombre y la obra”, en ARANGUREN, José Luis Id.: Obras completas, vol. 1, Madrid, Trotta.

CALVO SERER, Rafael (1953): La configuración del futuro, Rialp, Madrid.

CALVO SERER, Rafael (1953), "La politique interieur dans l'Espagne de Franco", en Écrits de Paris IX.

38 Lapesa, R. (1986), "Recuerdo y lección del 'plan Morente"”, Revista de Occidente núm. 60, 1986, pág. 81. 
DÍAZ DÍAZ, Gonzalo (1991): Hombres y documentos de la Filosofía española, vol. IV, CSIC, Madrid.

DÍAZ HERNÁNDEZ, Onésimo (2008): Rafael Calvo Serer y el grupo Arbor, Universitat de València, Valencia.

HERMIDA DEL LLANO, Cristina (1997): J.L.L. Aranguren, Estudio sobre su vida, obra y pensamiento, Universidad Carlos III-Dykinson, Madrid.

HERMIDA DEL LLANO, Cristina (1997) Aranguren (1909-1996), Ediciones del Orto, Madrid.

LAPESA, Rafael (1986): “Recuerdo y lección del 'plan Morente"”, en Revista de Occidente núm. 60, pp. 77-88,

MARÍAS, Javier (1988, 1994 y 1999): El País "Nada importa" (18/05/88), "El padre" (16/06/94), "El artículo más iluso" (26/06/99).

MARÍAS, Julián (2008): Una vida Presente. Memorias, Páginas de espuma, Madrid.

MAÑACH, Jorge (1958): "'El intelectual tiene que atender a lo social, pero sin comprometer su independencia', dice J. L. López Aranguren”, en Diario de la Marina, 14/12/1958.

MORENO PESTAÑA, José Luis (2013): La norma de la filosofía. La configuración del patrón filosófico tras la Guerra Civil, Biblioteca Nueva, Madrid.

MUGUERZA, Javier (1993) "Del aprendizaje al magisterio de la insumisión. Encuentro con José Luis L. Aranguren”, en LÓPEZ ARANGUREN, Eduardo, MUGUERZA, Javier y VALVERDE, José María Id.: Retrato de José Luis L. Aranguren, Galaxia GutembergCírculo de lectores, Madrid, pp. 65-88.

ROYO, Rodrigo (1965): "Las incógnitas éticas del profesor Aranguren”, en Revista SP, 15/06/1965.

SABORIT, Andrés (1961): Julián Besteiro, Impresiones Modernas S.A., México D.F.

VÁZQUEZ GARCÍA, Francisco (2009): La Filosofía española. Herederos y Pretendientes. Una lectura sociológica (1963-1990), Abada Editores, Madrid.

VV.AA. (2009): Aranguren. Filosofía en la vida y vida en la filosofía, Ministerio de Cultura, Madrid. 